\title{
Real-time measurement of Xenon in a binary gas mixture using ultrasound time-of-flight: a feasibility study
}

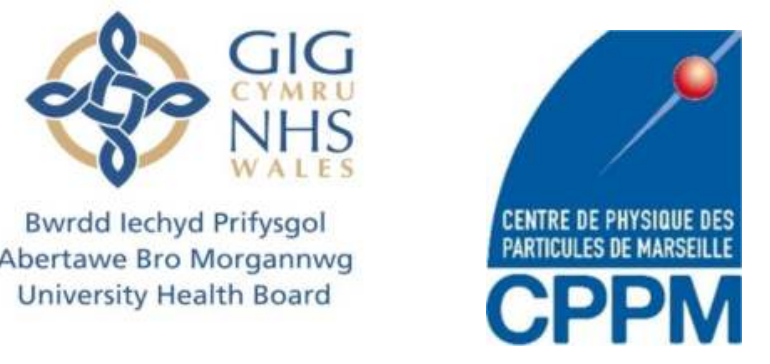

Williams DJ, ${ }^{1}$ Hallewell G, ${ }^{2}$ Chakkarapani E, ${ }^{3}$ Dingley J. ${ }^{4}$

${ }^{1}$ ABMU NHS Trust, Morriston Hospital, Swansea SA6 6NL, UK

${ }^{2}$ Centre de Physique des Particules de Marseille, Marseille 13288, France

${ }^{3}$ St. Michael's Hospital Bristol, Dept of Neonatology, Bristol, UK

${ }^{4}$ Swansea University, Swansea SA2 8PP, UK

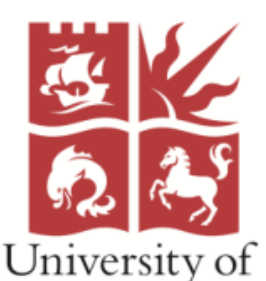

BRISTOL
Swansea University Prifysgol Abertawe

\section{Background}

Xenon $(\mathrm{Xe})$ is increasingly used in anaesthesia with $\mathrm{O}_{2}$ as a balance gas. Fractional concentrations of these gases are typically measured using a thermal mass meter (TMM) and fuel cell respectively.[1] An ultrasonic flow meter (UFM) measures volumetric flow of gas by measuring the difference in the time of flight (ToF) of a series of ultrasonic pulses propagating in and against the direction of gas flow.

Speed of sound $(c)$ in a binary gas mixture is related to temperature, pressure, and molar masses of the component gases.[2] (Fig. 1). Therefore a UFM could potentially be adapted to make a compact, non-invasive monitoring device which could measure both volumetric flow and $\mathrm{Xe}$ concentration (Xe\%). We built apparatus to derive $\mathrm{Xe} \%$ from a commercially available UFM $\left(\mathrm{Xe}_{\mathrm{UFM}}\right)$, and compared the results with simultaneous measurements from the gold standard of a TMM $\left(\mathrm{Xe}_{\mathrm{TMM}}\right)$.

Fig. 1

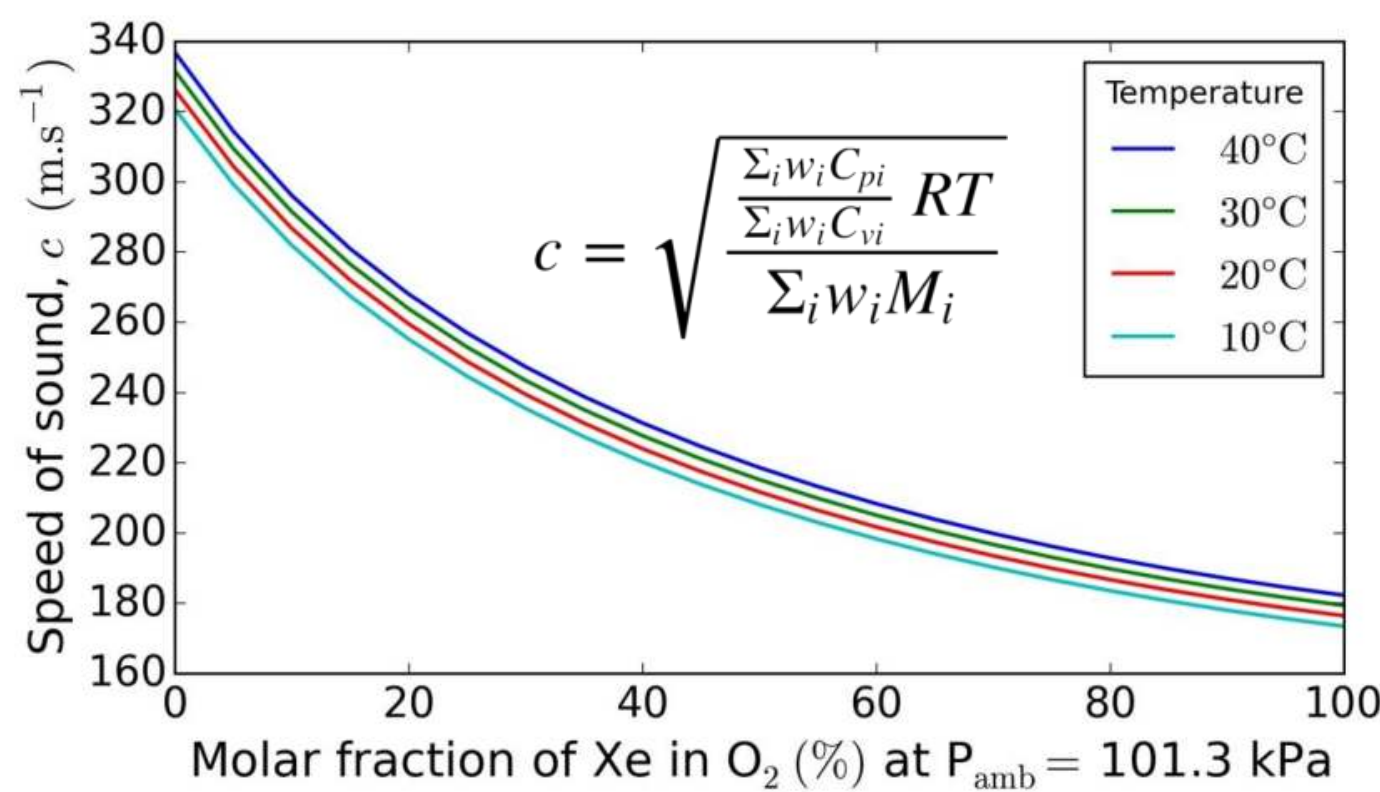

Where $w$ is molar fraction of each component gas $i ; C_{p}$ and $C_{v}$ are molar specific heat at constant pressure and volume $\left(\mathrm{J} \cdot \mathrm{mol}^{-1} \cdot \mathrm{K}^{-1}\right) ; R$ is molar gas constant $; T$ is absolute temperature $(\mathrm{K}) ; M$ is molar mass $\left(\mathrm{kg} \cdot \mathrm{mol}^{-1}\right)$

Method

A precision temperature sensor (LM35Z, Texas Instruments Inc., Dallas) was mounted in a short length of connector tubing with epoxy resin. A bespoke data logger designed around a microcontroller (Arduino Mega 2560) and associated hardware was used to coordinate: 10-bit Analogue-to-Digital conversion (ADC) and rolling-mean smoothing of the output from the temperature sensor; two-way serial communication with the UFM (Spirocell ${ }^{\mathrm{TM}}$, Gill Ltd, UK) and TMM (GKM-03, INSOVT, Russia); parsing of all data streams; and calculation of $\mathrm{Xe}_{\mathrm{UFM}}$ using a look-up table of concentration vs. temperature-corrected speed of sound with linear interpolation. Current time, temperature, $\mathrm{ToF}$ measurements, $\mathrm{Xe}_{\mathrm{UFM}}$ and $\mathrm{Xe}_{\mathrm{TMM}}$ readings were displayed in real time to two decimal places of accuracy on a backlit LCD display. All data were simultaneously saved to a micro SD card and transmitted via Bluetooth to a laptop computer as .csv spreadsheet files for subsequent analysis (Fig. 2).

Fig. 2

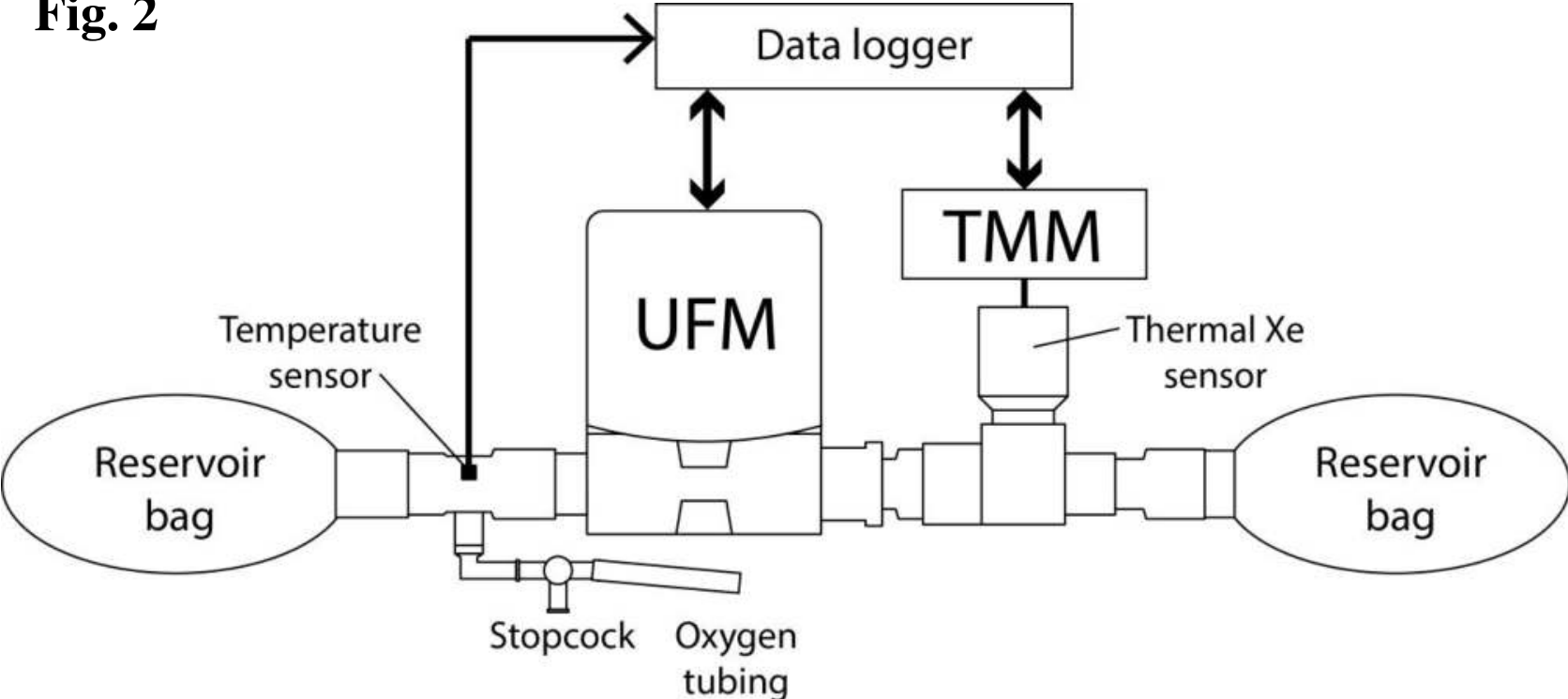

Two point calibration of the UFM, TMM, and temperature sensor was performed with the apparatus at sea level $\left(\mathrm{P}_{\mathrm{amb}}=101.3 \mathrm{kPa}\right)$. The apparatus was purged with $100 \%$ Xenon via the stopcock. The two reservoir bags were alternately and repeatedly inflated and deflated to mix gas throughout the system until $\mathrm{Xe}_{\mathrm{TMM}}$ was constant and $>98 \%$.

When movement of gas had ceased and $\mathrm{Xe}_{\mathrm{TMM}}$ was stable, the reservoir bags were clamped and 20 consecutive paired measurements of $\mathrm{Xe}_{\mathrm{UFM}}$ and $\mathrm{Xe}_{\mathrm{TMM}}$ were taken at $2 \mathrm{~s}$ intervals using the data logger. The clamps were removed. $20 \mathrm{~mL}$ aliquots of gas were aspirated and replaced with $100 \% \mathrm{O}_{2}$ via the three-way stopcock to reduce $\mathrm{Xe}_{\mathrm{TMM}}$ in approximately $0.5 \%$ increments. The gas was mixed and a further set of 20 readings were taken as above. This procedure was repeated until $\mathrm{Xe}_{\mathrm{TMM}}<5 \%$.

The paired readings for $\mathrm{Xe}_{\mathrm{UFM}}$ and $\mathrm{Xe}_{\mathrm{TMM}}$ were compared using a scatter plot and Bland-Altman (BA) analysis.

\section{Results}

2,029 data pairs of $\mathrm{Xe}_{\mathrm{UFM}}$ and $\mathrm{Xe}_{\mathrm{TMM}}$ were collected. Linear regression analysis of the scatter plot showed that mean values for $\mathrm{Xe}_{\mathrm{UFM}}$ correlated closely with $\mathrm{Xe}_{\mathrm{TMM}}$ across the range $5 \%$ to $95 \%(\mathrm{r}=0.989$; linear fit equation: $\left.X e_{U F M}=1.108 * X e_{T M M}-3.528\right)$. However BA analysis (Fig. 3) showed that variability of individual readings and non-linearity increased as $\mathrm{Xe}$ concentration increased to an extent which was unsuitable for clinical use.
Fig. 3

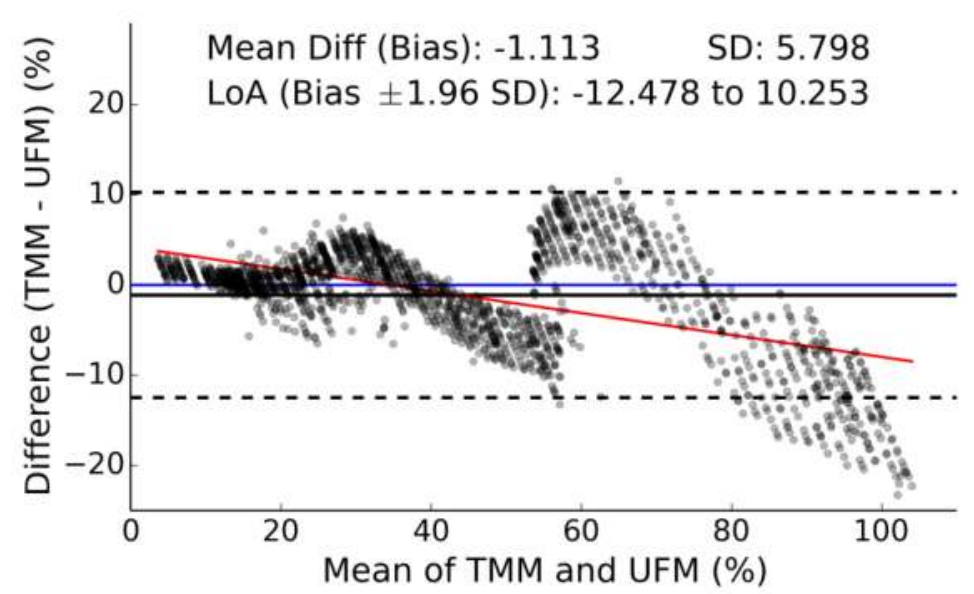

Fig. 4

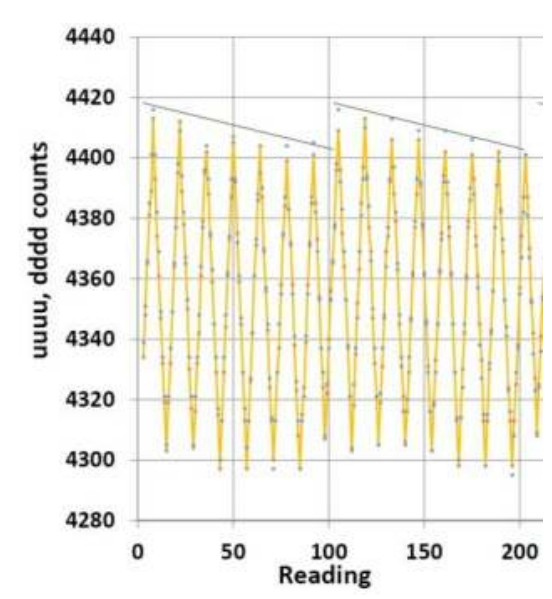

Subsequent studies using an oscilloscope and high frequency data capture showed that the variability was caused by the hardware and firmware of the UFM, which applied different levels of gain in a bimodal saw tooth pattern to 128 successive ToF measurents over a $1.28 \mathrm{~s}$ period (Fig. 4). This effect could be corrected by time-averaging. Non-linearity may be due to echoes and phase cancellation effects resulting from the 'pinched reflex' geometry of the angled acoustic mirrors used in the UFM. Further studies are in progress to characterise and compensate for these effects.

\section{Conclusions}

A commercially available ultrasonic flow meter (UFM) can be used to estimate real-time $\mathrm{Xe} \%$ in a binary mix with $\mathrm{O}_{2}$. With further work, it may be possible to adapt an existing commercial UFM, or design a bespoke UFM to non-invasively perform both volumetry and noninvasive binary gas concentration measurement to a clinically acceptable degree of accuracy and precision.

\section{References}

[1] Lynch C et. al. Xenon anesthesia. Anesthesiol. 2000;92(3):865-8

[2] Bates R et. al. Implementation of ultrasonic sensing for high resolution measurement of binary gas mixture fractions.

Sensors 2014, 14:11260-76

[3] Lemmon, E Huber H, McLinden M. REFPROP Standard Reference Database 23, version 9.0; National Institute of Standards and Technology: Gaithersburg and Boulder, MD, USA, 2010 